Supporting information to accompany

\title{
"Structured Assemblies of Ferromagnetic Particles through covalent Immobilization on Functionalized Polymer Surfaces obtained by Surface Segregation"
} Antoine Bousquet, ${ }^{1}$ Emmanuel Ibarboure, ${ }^{1}$ Christine Labrugère, ${ }^{2}$ Eric Papon, ${ }^{1}$ Juan Rodriguez-Hernandez ${ }^{1}$ *

${ }^{1}$ Laboratoire de Chimie des Polymères Organiques (LCPO-UMR 5629), ENSCPB, University Bordeaux 1, 16, Avenue Pey Berland, 33607 Pessac-Cedex, France

${ }^{2}$ Institut de Chimie de la Matière Condensée de Bordeaux (ICMCB-CNRS), 87, Avenue du Docteur Schweitzer 33608 Pessac-Cedex, France

\section{a) Materials}

Monomers and solvents. The monomers (styrene and tert-butyl acrylate) were dried over $\mathrm{CaH}_{2}$ and distilled under reduced pressure. The solvents were used as received. The magnetic particles were supplied by Ademtech S.A. (www.ademtech.com). The particles are monodisperse with an average size of $200 \mathrm{~nm}$ and consist of organic ferrofluid calibrated droplets covered by a thermoplastic layer functionalized with amine groups. The amount of primary amine has been estimated to be $120 \mu \mathrm{mol} / \mathrm{g}$ of dried particle by colorimetric measurements using TNBS (Trinitrobenzene sulfonic acid).

\section{b) General methods}

${ }^{1} \mathrm{H}$ NMR spectra of the copolymers were recorded at room temperature on a Bruker Avance $400 \mathrm{MHz}$ spectrometer using the residual proton resonance of the deuterated solvent as an internal standard. Average molar masses and molar mass distributions of the samples were determined by Size Exclusion Chromatography (SEC), using a Varian 9001 pump with both a refractive index (Varian RI-4) and a UV detector (Spectrum Studies UV 150). Calibration was 
obtained using narrowly-distributed polystyrene standards and THF as the mobile phase at a flow rate of $0.5 \mathrm{ml} \cdot \mathrm{min}^{-1}$.

Contact Angle Measurements. Contacts angles of water were determined using a Krüss DSA100 contact angle measuring system at room temperature. A water droplet of $3 \mu$ was placed on the specimens at two different $\mathrm{pH}$ values: 2.6 and 7 . A charge coupled device camera was used to capture the images of the water droplets for the determination of the contact angles.

X-ray Photoelectron Spectroscopy (XPS) Experiments. XPS spectra were recorded with a 220i-XL ESCALAB from VG. The films supported on silicon wafer were put under UHV to reach the $10^{-8} \mathrm{~Pa}$ range. The Non-monochromatized $\mathrm{Mg} \mathrm{X}$-ray source was used at $100 \mathrm{~W}$, as well as a flood gun to compensate for the nonconductive samples. The spectra were calibrated in relation to the C1s binding energy $(284.6 \mathrm{eV})$, which was applied as an internal standard. Fitting of the high-resolution spectra was provided through the AVANTAGE program from VG.

Atomic Force Microscopy (AFM) images were recorded in the air with a Nanoscope IIIa microscope operating in tapping-mode (TM). The probes were commercially available silicon tips with a spring constant of $42 \mathrm{~N} / \mathrm{m}$, a resonance frequency of $285 \mathrm{kHz}$, and a typical radius of curvature in the $10-12 \mathrm{~nm}$ range. Both the topography and the phase signal images were recorded with a resolution of $512 \times 512$ data points. Samples for atomic force microscopy analysis (AFM) were solvent-cast at room temperature using $1 \mathrm{wt} \%$ THF solutions.

\section{c) Synthesis of the block copolymers}

The diblock copolymers have been prepared by ATRP in two steps following previously reported procedures. The synthesis is briefly described below: 
Synthesis of polystyrene (PS) macroinitiator by ATRP. All polymerizations were performed in schlenks previously flamed and dried under vacuum. ATRP was carried out using the following initial stoichiometrie $[\mathrm{M}]:[\mathrm{I}]:[\mathrm{CuBr}]:[\mathrm{L}]=250: 1: 1: 2$, where $\mathrm{M}=$ styrene, $\mathrm{I}=$ initiator $(\mathrm{PhEBr})$ and $\mathrm{L}=$ ligand (bipy). The reactants were added under $\mathrm{N}_{2}$. The reaction mixtures were then degassed by three freeze-pump-thaw cycles and placed in a thermostated oil bath at $110^{\circ} \mathrm{C}$. After the polymerization, the mixtures were cooled to room temperature; the contents were diluted with dichloromethane $\left(\mathrm{CH}_{2} \mathrm{Cl}_{2}\right)$ and passed through a neutral alumina column to remove the copper salt. After evaporation, the polymers were precipitated in ethanol, filtered, washed and dried under vacuum.

Synthesis of PS-b-PtBA. The macroinitiators either PS-Br or PnBA-Br and $5 \mathrm{~mL}$ of degassed acetone were added to the mixture $([\mathrm{M}]:[\mathrm{I}]:[\mathrm{CuBr}]:[\mathrm{L}]=400: 1: 1: 1)$. Acetone enhanced the solubility of the $\mathrm{CuBr} / \mathrm{PMDETA}$ complex. The $t \mathrm{BA}$ polymerizations were carried out at $65^{\circ} \mathrm{C}$.

Hydrolysis of the PtBA block in the PS-b-PtBA copolymer. Copolymers were first dissolved in $\mathrm{CH}_{2} \mathrm{Cl}_{2}$. Trifluoroacetic acid (TFA) was then added (10 equivalent to $t$-butyl ester units), and the mixture was stirred at room temperature for 3 days. The deprotected polymers precipitated in the reaction media, were filtered and washed with $\mathrm{CH}_{2} \mathrm{Cl}_{2}$ and finally dried under vacuum.

The GPC traces of PS precursor, the diblock copolymer PS-b-PtBA and the ${ }^{1} \mathrm{H}-\mathrm{NMR}$ spectra of PS precursor, PS-b-PtBA and PS-b-PAA are depicted in Figures S1 and S2 respectively. 


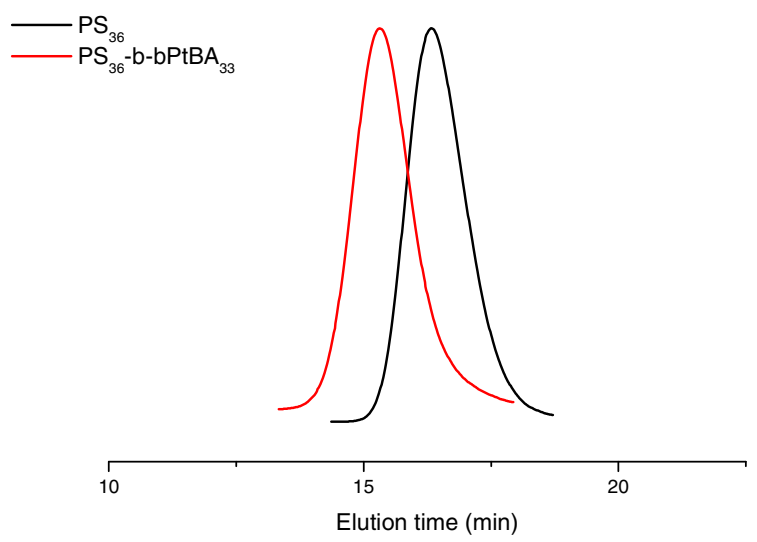

Figure S1.- GPC traces of $P S_{36}$ precursor, the diblock copolymer $P S_{36}-b-P t B A_{33}$.

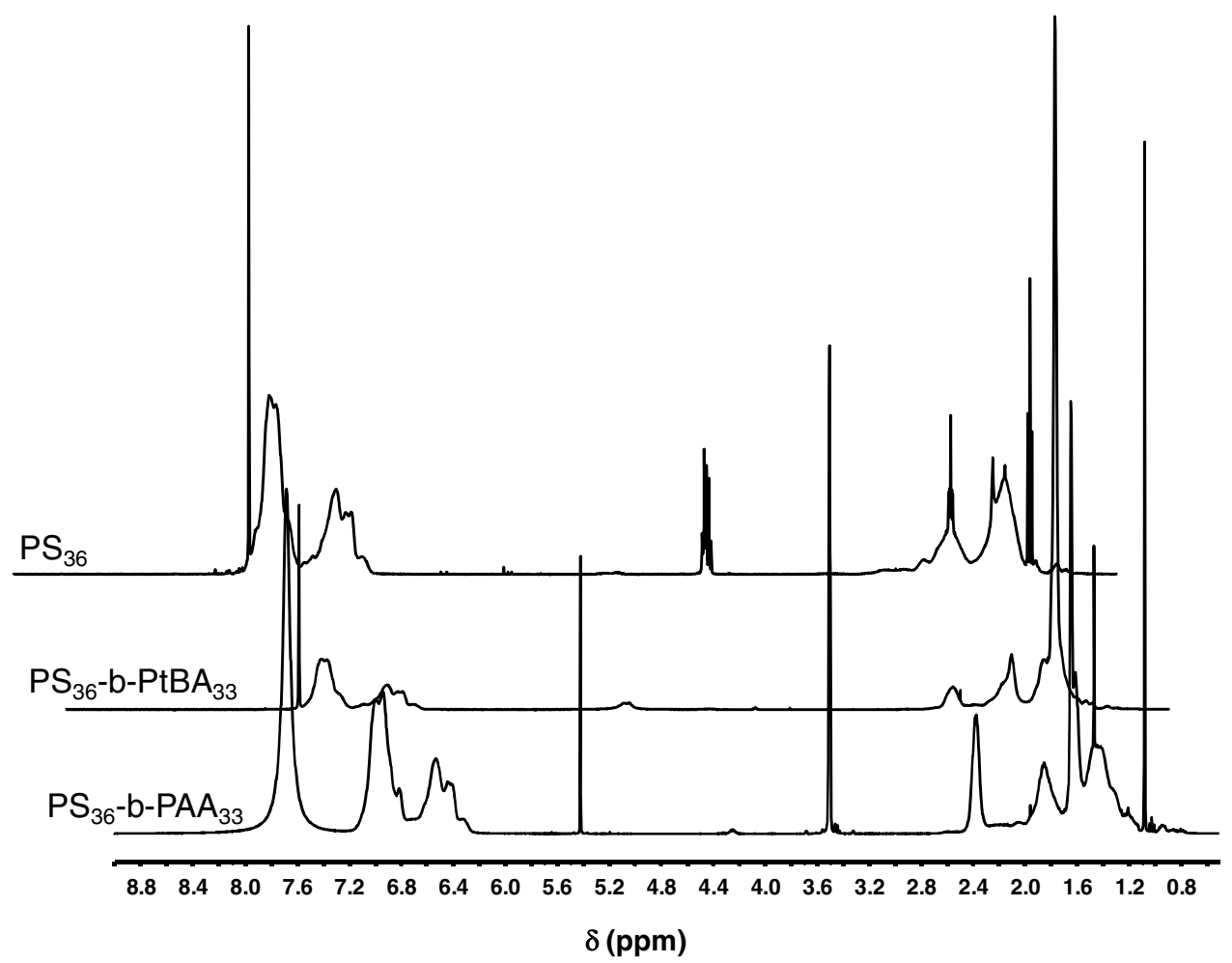

Figure S2.- ${ }^{I} H-N M R$ spectra of $P S_{36}$ precursor, $P S_{36}-b-P t B A_{33}$ and $P S_{36}-b-P A A_{33}$.

\section{d) Surface migration and characterization of the surfaces}

A mixtures containing between $10-40 \%$ of diblock copolymer $\left(\mathrm{PS}_{36}-\mathrm{b}-\mathrm{PAA}_{33}\right)$ and $90-60 \%$ of a linear polystyrene respectively (Mw: $74000 \mathrm{~g} / \mathrm{mol}$ ) were dissolved in THF (concentration: 
$10 \mathrm{mg} / \mathrm{ml}$ ). The solution was spin coated on a cleaned silicon wafer and annealed during 4 days at $90^{\circ} \mathrm{C}$ in a humid atmosphere. After the treatment, the surface was dried under vacuum. The films were characterized both by contact angle measurements and X-ray photoelectron spectroscopy using in all cases, for comparative purposes, a PS film that has followed the same treatment. Whereas the diblock copolymer exhibits very low contact angles that demonstrate the surface rearrangement (see Figure 3) the PS film remains hydrophobic.

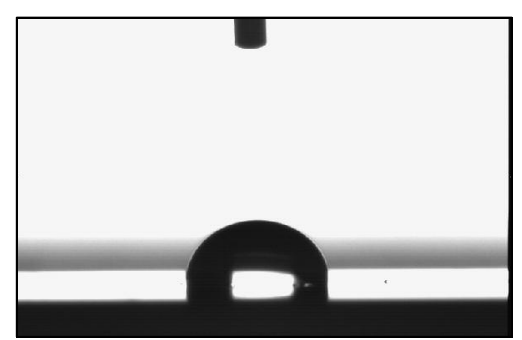

(i)

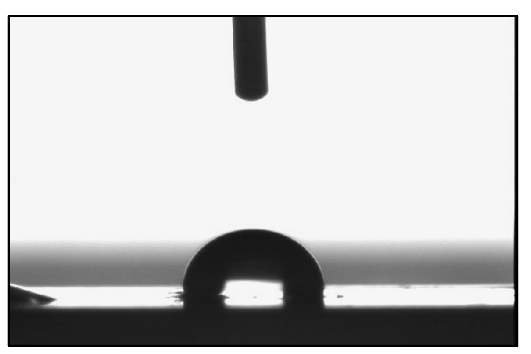

(ii)

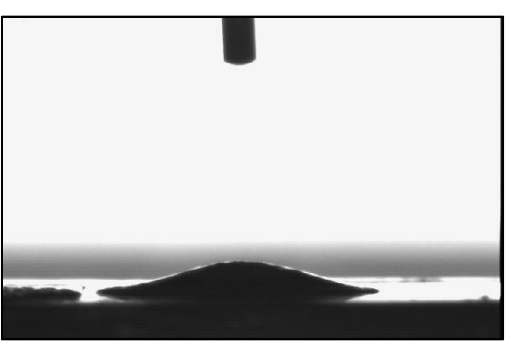

(iii)

Figure S3. Contact angle measurements carried out on three different samples: (i) PS used as reference, (ii) diblock copolymer blend before treatment, (iii) diblock copolymer blend after thermal treatment in humid environment.

The XPS spectra exhibit a large relative increment of the oxygen signal associated to the migration of the PAA block to the surface (Figure S4). The composition-depth profile of the sample exposed to water confirmed the difference between the surface composition and the overall bulk composition. After the analysis of the outmost polymer layer a large surface excess of the PAA segment, 5.5\%, was observed which is gradually reduced down to $1 \%$ in the bulk. 

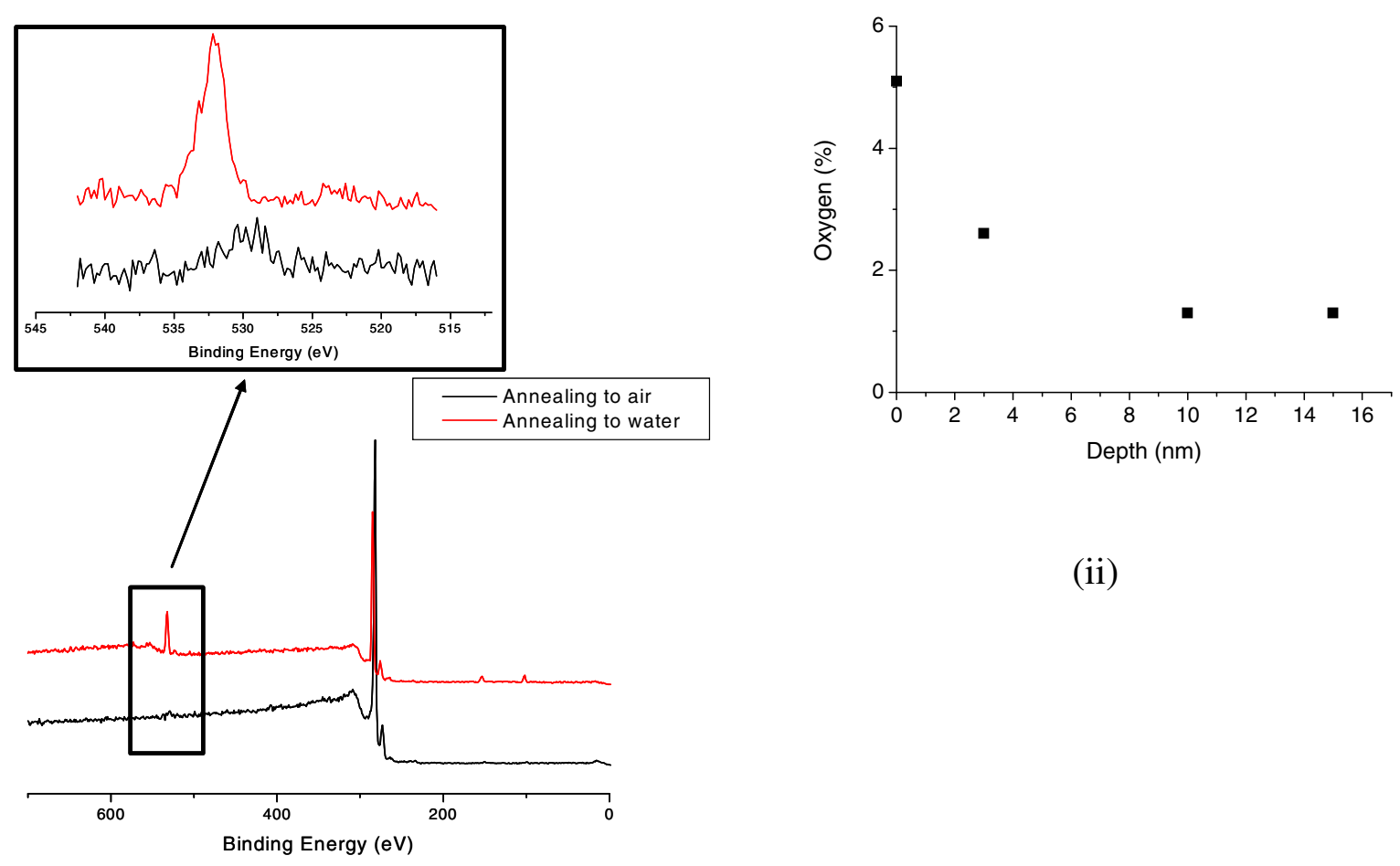

(ii)

(i)

Figure S4.- XPS spectra of (i) blend containing 20\% of diblock $P S_{36}-b-P A A_{33}$ and $80 \%$ of PS water and air annealed and (ii) Composition-Depth profile for the sample annealed to water.

Load and release of methylene blue (MB) (Figure S5): the films annealed to water vapour supported on a silicon wafer were submerged in a buffered MB solution at $\mathrm{pH} 7.0$ with a concentration of $\mathrm{MB}$ of $10^{-3} \mathrm{M}$. Note that at neutral $\mathrm{pH}$ values PAA is negatively charged and can establish electrostatic interactions with the positively charged MB (loading step). After 1 h. the films were rinsed in a separate bath at $\mathrm{pH} 7.0$ during 30 minutes thereby removing the $\mathrm{MB}$ in excess. Finally, the release of the MB was carried out by dipping the films into an acidic water solution ( $\mathrm{pH}$ 3.0). The quantity of $\mathrm{MB}$ released was estimated by UV spectroscopy (Lambert-Beer's Law). Figure S5 depicts schematically the procedure followed to quantify the amount of carboxylic functions at the interface for water annealed surfaces. 
1) Load:

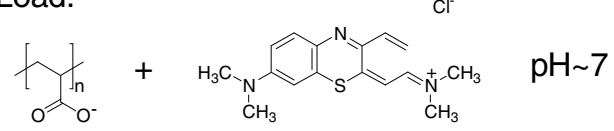

2) Rinse:
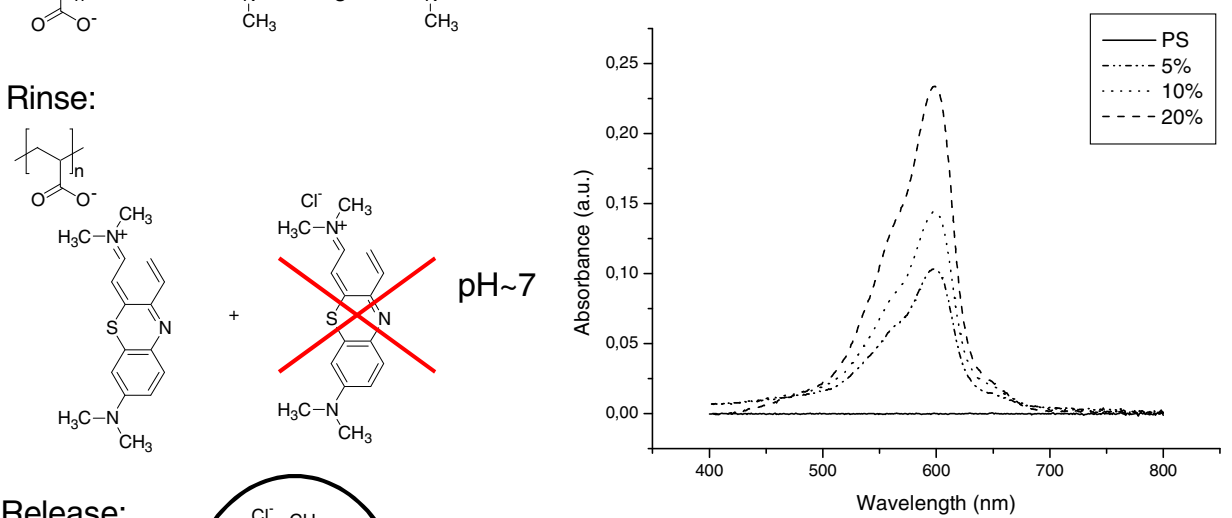

3) Release:

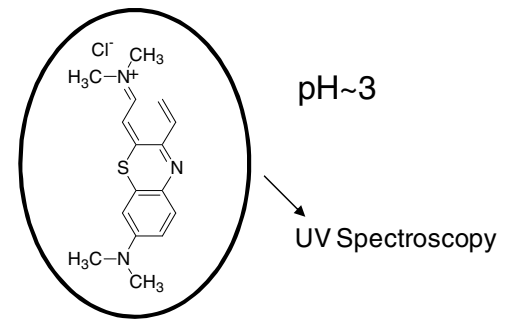

Figure S5. General scheme followed for the load and release of MB. On the right: UV-vis spectra obtained for polymer blends treated to water vapour having different diblock $P S_{36}-b-P A A_{33}$ percentage within the blend (5, 10 and $20 \%)$

\section{e) Covalent attachment of the surface aminated magnetic particles}

The carboxylic groups revealed at the surface were employed to covalently attach the magnetic particles that are enrobed of amine functions at the surface. For that purpose, we have activated the acidic groups using a water-soluble carbodiimide: N-cyclohexyl-N'-(2morpholinoethyl)-carbodiimide methyl-p-toluene-sulphonate (CMTS). The films were submerged into $20 \mathrm{ml}$ of a water solution $(\mathrm{pH} 7)$ and a large excess of CMTS was added to activate in a first step the carboxylic functions. Then, $0,2 \mathrm{ml}$ concentrated solution of the particles $(1 \%)$ were added to the activated surface. The concentration of the particles in the final solution was $0.01 \%$. The reaction proceeds overnight at room temperature. Those nongrafted particles that have been adsorbed to the surface by electrostatic interactions between the negatively charged carboxylic functions and the positive amine groups were removed by extensive washing with an acidic solution. Note that, at low $\mathrm{pH}$ values, whereas the carboxylic functions are protonated and loss the charge, the amine groups still positively charged and favoured the solubility of the particles. For comparative purposes, we repeat the rinsing procedure on similar films prepared by using the same conditions in which, on the 
other hand, no carbodiimide has been employed. In this case, the particles, which are exclusively adsorbed, were easily removed with water at low $\mathrm{pH}$ values.

\section{f) Organization of the particles as a function of the magnetic field}

The grafting of the particles has been carried out both in the presence or in the absence of a permanent magnetic field with a similar setup to the one depicted in Figure S6. A slow agitation was maintained to assure the colloidal stability without decantation of the particles. In the experiments performed with the magnet $(8 \mathrm{mT})$ the field lines were previously determined with iron and the magnet was placed below the solution.

(i)

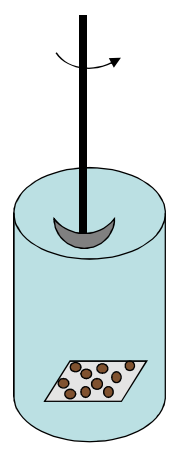

(ii)

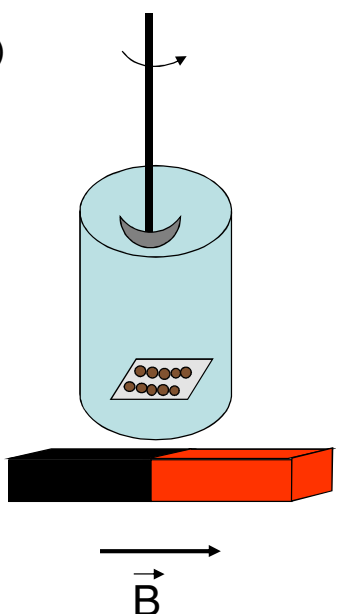

Figure S6.- Configuration setup for the orientation of the magnetic particles using a permanent magnet. 
g) Additional AFM images of the covalently attached magnetic particles

g.1) Phase and height images of the non-oriented particles:
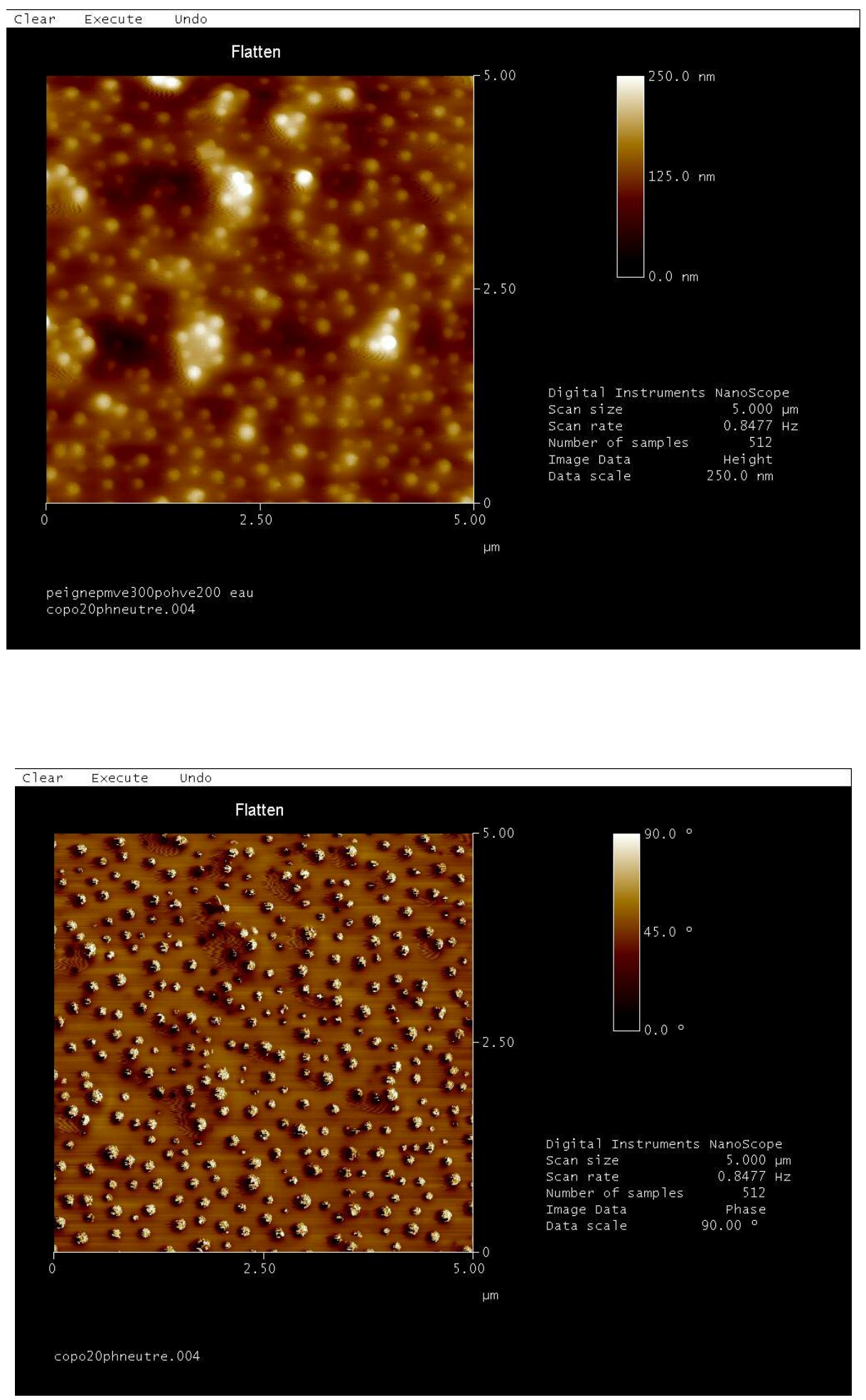
g.2) Phase and height images of the oriented particles:
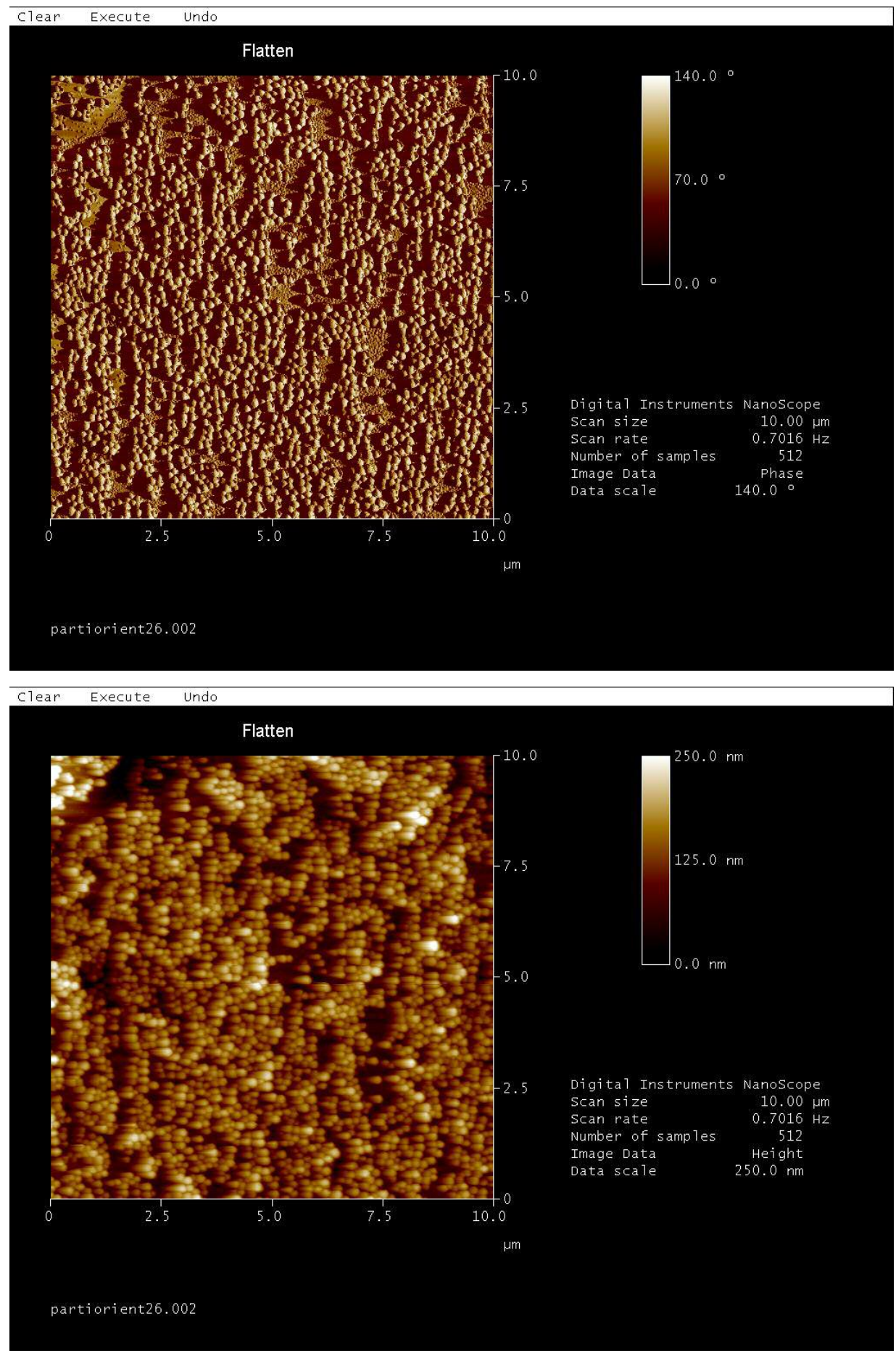